\section{Genetic and Metabolic Disease in Pediatrics}

Butterworth Internationai Medical Reviews, Pediatrics Volume 5. Edited by June $\mathrm{K}$ Lloyd and Charles R Scriver. (Pp 324; figures + tables. $£ 45 \cdot 00$.) London: Butterworth. 1985.

Most genetic and metabolic diseases are apparent at birth, are detectable before or soon after birth, or become evident during childhood. There are important exceptions which do not become evident until adulthood.

In this book inherited structural and functional disorders have been chosen by the editors and their 14 contributors to illustrate the interplay of genotype and environment which produces the many and varied disease phenotypes described in paediatric practice. Although some of the examples are individually rare this has been skilfully achieved by keeping homeostasis as a main theme.

In an excellent introduction the editors explain in a chapter entitled 'Genetic causes of deviant metabolism. What do we know? What can we do?' their reasons for choosing the subsequent 11 chapters to illustrate their homeostatic theme. Marcus Pembrey and Robert S Sparkes, in workmanlike and lucid accounts of genes, their anatomy, biochemistry, function, and anomalies, provide for the nongeneticist a helpful introduction to the language and techniques of the geneticist. In chapter 4 , Byers and Bonadio give a fascinating and up to the moment account of the molecular basis of clinical heterogeneity in osteogenesis imperfecta by reference to studies of inherited disorders of type I collagen. The next four chapters deal with the molecular basis for abnormal lysosomal enzyme transport in mucolipidoses II and III, the lactic acidaemias, defects in urea synthesis, and the hyperphenylalaninaemias. All give excellent individual accounts of the disorders and illustrate how such defects affect the body's homeostatic mechanisms. These chapters, together with those on the hyperlipidaemias, maternal-fetal metabolic homeostasis, maternal phenylketonuria, and androgen receptor disorders, well illustrate the intricate interplay between environment and genetic endowment in the maintenance of constancy in the body's 'milieu interior'.

I can recommend this volume to all geneticists with an interest in congenital abnormalities and metabolic disorders and all paediatricians who would wish to understand better their genetic basis, together with the biochemists who deal with metabolic disorders, geneticists, and paediatricians. This "evolutionary view of disease in man" raises issues beyond those of prevention and therapy of specific disorders, including moral and social issues. For these reasons I would recommend this book to all who wish to understand better the basis for a healthy life in metabolic equilibrium and the function, if not the origin, of our species.

FORRESTER COCKBURN

\section{An Introduction to Recombinant DNA}

By A E H Emery. (Pp x + 223; figures + tables. f6.75.) Chichester: Wiley. 1984.

Doctors are notorious for using two long words when a single short one would suffice. Molecular biology has gone even further by devising a new language of its own, which for the uninitiated makes a difficult subject almost incomprehensible. Thus, it is a pleasure to encounter a book which removes much of the mystery and mystique from a subject which promises to revolutionise medicine.

This lucid and concise text covers all aspects of molecular genetics in 10 short succinct chapters. The first sets the scene with an historical background and is followed by an outline of the structure of DNA. The technology for handling DNA is covered in chapter 3 while chapter 4 moves on to a consideration of the structure and function of the genome.

Chapters 5 and 6 are devoted to a review of the molecular pathology of Mendelian and multifactorial disorders and, in conjunction with chapter 7 which discusses the existing and potential applications of these techniques in clinical genetics, will be of particular interest to those involved in genetic counselling. The concluding chapters provide an overview, occasionally speculative, of the possible applications of the 'new genetics' in the broader fields of gene therapy, agriculture, and the study of evolution. The moral and ethical aspects of these developments are also considered.

Each chapter is well illustrated with line drawings and is followed by an appropriate bibliography. The book is completed by a particularly valuable glossary and comprehensive index. This well written and remarkably inexpensive book will be much appreciated by students and practitioners of all branches of medicine and can be highly recommended. This will undoubtedly be the first of many editions.

I D Young

Basic Cloning Techniques. A Manual of Experimental Procedures

Edited by R H Pritchard and I B Holland. (Figures + tables. $£ 11 \cdot 80$.) Oxford: Blackwell Scientific Publications. 1985.

There is currently no shortage of practical textbooks 